\title{
A CROSS-LAYER SCHEDULER WITH CHANNEL STATE INFORMATION FOR GUARANTEED QUALITY OF SERVICE
}

\author{
${ }^{1}$ R. Nandhini and ${ }^{2}$ N. Devarajan \\ ${ }^{1}$ Department of Computer Science Engineering, Anna University, Chennai, India \\ ${ }^{2}$ Department of Electrical and Electronics Engineering, Government College of Technology, Coimbatore, India
}

Received 2013-09-18; Revised 2013-10-23; Accepted 2013-11-13

\begin{abstract}
WiMAX networks are deployed for commercial use because of its nature high bandwidth. This requirement invokes the application level changes in QoS provisioning techniques. The objective of the broadband wireless technologies is to ensure the end to end Quality of Service (QoS) for the service classes. WiMAX is a revolution in wireless networks, which could support real time multimedia services. In order to provide QoS support and efficient usage of system resources an intelligent scheduling algorithm is needed. The design of the detailed scheduling algorithm is a major focus for researchers and service providers. In this study, a channel aware cross-layer scheduling algorithm for WiMAX networks has been proposed. This scheme employs the Signal to Noise Ratio (SNR) value, which allocates the bandwidth based on the information about the quality of the channel and the service requirements of each connection. The proposed algorithm is described in detail and evaluated, through a series of simulations. The QoS parameters of throughput, bandwidth efficiency and transmission efficiency have been measured in simulation.
\end{abstract}

Keywords: Cross-Layer, IEEE 802.16, QoS, Scheduling, WiMAX

\section{INTRODUCTION}

World Wide Interoperability for Microwave Access (WiMAX) is an IEEE standard (IEEE 802.16d/e) that promises high bandwidth solution with long range for metropolitan area networks. IEEE 802.16 is able to cover large geographical area since the distance between the Base Station (BS) and the Subscriber Station (SS) can extend up to 30 miles. IEEE 802.16 defines the layer 1 (Physical (PHY)) and layer 2 (Data link or Media Access Control (MAC)) of the Open System Interconnection (OSI) seven layer network model. The different types of standards for PHY supports are Single Carrier (SC), Single Carrier Access (SCA), Orthogonal Frequency Division Multiplexing (OFDM) and Orthogonal Frequency Division Multiple Access (OFDMA). Recent researches focus mainly on the OFDM and OFDMA PHY supports. These standards define two operational modes for communication namely; mesh mode and point-tomultipoint mode. In mesh mode, the SSs can communicate with each other and also with the BS. In point-to-multipoint mode, SSs are supposed to communicate only through BS. BS has dedicated buffers and slots for downlink connection. During uplink, slots are allotted per SS and not per connection. Uplink channel is shared by all SSs, whereas downlink channel is used only by BS.

IEEE 802.16e is expected to provide QoS for fixed and mobile users. QoS depends upon a number of implementation details like scheduling, buffer management and traffic shaping. The responsibility of scheduling and BW management is to allocate the resources efficiently based on the QoS requirement of the service classes.

There are five service classes which are defined in IEEE802.16e standard. They are as follows:

Corresponding Author: R. Nandhini, Department of Computer Science Engineering, Anna University, Chennai, India 
- Unsolicited Grant Services (UGS): Designed to support Constant bit rate services like voice applications

- Real Time Data Polling Services (RTPS): Designed to support real time services that generates variable size data packets on a periodic basis like MPEG but insensitive to delay

- Extended Real Time Polling Services (ERTPS): Designed to support real time applications with variable data rates which require guaranteed data and delay. Example: Voice Over Internet Protocol (VOIP) with silence suppression

- $\quad$ Non Real Time Polling Services (NRTPS): Designed to support non real time and delay tolerant services that require variable size data grant burst types on a regular basis such as File Transfer Protocol (FTP)

- Best Effort (BE): Designed to support data streams that do not require any guarantee in QoS such as Hyper Text Transfer Protocol (HTTP)

The QoS provision in WiMAX requires complete scheduling mechanism which is not defined in the standard. The scheduling mechanisms have to provide guarantee to the bandwidth required by SS as well as wireless link usage. The goal of designing a scheduler is to minimize power consumption and Bit Error Rate (BER) and to maximize the total throughput. Wired networks scheduling algorithms are unfit for wireless networks due to location dependency and burst channel errors. Thus, the scheduling algorithm should take WiMAX QoS classes and service requirements into consideration.

\subsection{Literature Review}

Borin and Fonseca (2009) proposed a standard compliant scheduling solution for uplink traffic in IEEE 802.16 networks but wireless channel characteristics are not considered in this solution. Many other scheduling mechanisms have been proposed in the past research work. But none of them is able to support QoS requirements of the five types of service flow defined by the IEEE 802.16e standard.

It has been proved that the scheduling algorithm that considered wireless link perform better than the algorithm that does not consider the nature of the wireless link, delay and buffer size. Schedulers can use different metrics to estimate the channel condition. Ghazizzadeh et al. (2009) it is estimated according to the instantaneous transmission rate. Fluid Fair Queuing (FFQ) is a well-known algorithm which provides fairness among the packets through the shared link. Chandra and Praveen (2009) classified the uplink schedulers as Weighted Round Robin (WRR), Earliest Dead line First (EDF) and Weighted Fair Queuing (WFQ). Down link schedulers are classified into Proportional Fairness (PF), Adaptive Proportional Fairness (APF), Integrated Cross-Layer (ICL) and Round Robin (RR).

Revankar et al. (2010) the authors emphasis the MAC scheduling architecture for IEEE 802.16 wireless networks in both uplink and downlink direction to broadcast the frame. Further they used WFQ as uplink as well as downlink scheduling algorithm for improving delay and throughput. There is no separate scheduling policy for Unsolicited Grant Services (UGS). EDF is appropriate for real time data Polling Services (rtPS) WFQ for non-real time Polling Services (nrtPS). Remaining Bandwidth is split for all BE connections. This work has not considered extended real time Polling Services (ertPS). Cross layer communications would be needed to tell MAC layer about the transitions. They have not considered OFDMA scheduler and traffic classes. Even though there are vast number of works based on scheduling in single hop networks, these algorithms cannot be applied for multihop relay scenarios. A cross layer approach to packet scheduling in Mobile wimax has been emphasized by Shuaibu et al. (2010). They showed how the wireless link affects the throughputs and the frame utilization by using adaptive modulation and coding, the wireless link should be considered.

$\mathrm{Lu}$ and $\mathrm{Ma}$ (2011) proposed packet-scheduling algorithms for output-buffered switches which support Quality of Services (QoS) transmit packets in some priority order, e.g., according to dead-lines, virtual finishing times, eligibility times, or other time stamps that are associated with a packet. TCP aware uplink scheduling algorithm focuses on the allocation of bandwidth higher than actual sending rate of the connection. Comparative analysis of different QoS algorithm in WiMAX has been extensively studied in the past literatures. Wu et al. (2012) proposed the cross layer scheduler in which each down link is maintained using an output port manager. The output port manager implements mechanisms that support QoS such as buffer management and scheduling.

\subsection{IEEE 802.16 Scheduling Architecture}

The basic IEEE 802.16 architecture includes Base station and multiple Subscriber Stations (SS). Both 
base station and subscriber station are immobile when client wants to connect SS to a mobile station. Base station acts as a central entity which transfers all the data from the subscriber stations in point-to-multi point architecture. Two or more subscribers are not allowed to communicate directly. The BS and SS architecture are connected through wireless links. Communication occurs in two directions: From BS to $\mathrm{SS}$ is called downlink and from SS to BS is called uplink. During downlink, BS broadcasts data to all subscribers and subscriber selects packets destined for it. Uplink channel is shared between all multiple SSs while downlink channel is used only by BS. Figure 1 depicts the basic architecture of IEEE 802.16.

In order to ensure slotted channel sharing and the slots are allocated by BS to various SS in one uplink frame, Time Division multiplexing (TDD) or Frequency Division multiplexing (FDD) is used. This slot allocation information is broadcast by BS through the Uplink Map message (UL-MAP) at the beginning of each frame. ULMAP contains information element which includes the transmission opportunities and the time slots in which the SS can transmit during the uplink subframe.

\subsection{Scheduling Algorithms}

IEEE 802.16 MAC layer adopts a connection oriented architecture in which a connection must be established before data communications. Each connection is assigned a unique identifier (connection ID) and it is associated with a service flow which defines the desired QoS level of the connection. In a standard scheduling framework, data packets arriving at the BS are classified into connections which are then classified into service flows. Packets of same service flow are placed in a queue and then further classified based on their service priorities of the connection. For packets in multiple queues with different service requirements, a packet scheduler is employed to decide the service order of the packets from the queues. If properly designed a scheduling algorithm may provide the desired service guarantees.

The scheduler should consider the following important parameters:

- The traffic service type

- The set of QoS requirements of the connections

- The capacity of bandwidth for data transmission
- The bandwidth requirements from the connections

- Waiting time of bandwidth request in the system

The ideal scheduler should be able to make optimum use of the available bandwidth to reduce traffic delays and satisfy the QoS requirements to the best extent so as to reduce packets drop rate and sustain the QoS support.

WiMAX schedulers can be classified into two main categories, channel unaware schedulers where the channels are assumed to be error free and channel aware schedulers where channel state information is taken into consideration while scheduling the packet. Channel unaware schedulers are further classified into homogeneous and hybrid schedulers. Hybrid schedulers combine more than one scheduler to satisfy the QoS requirements of the multiple service class traffic in WiMAX networks.

Figure 2 represents the cross-layer scheduler methodology. WRR, WFQ, EDF, Strict Priority (SP) are the few examples of homogeneous scheduling algorithms. According to the research, none of the homogeneous scheduling algorithm provides the QoS requirement of WiMAX networks. So, researchers attempted to hybrid the algorithms to get a satisfied QoS level. Cross-layer scheduling is one of the algorithms in channel aware scheduling algorithm.

\subsection{Proposed Cross-Layer Scheduling Algorithm}

The main focus of the cross layer design is to provide best possible end-to-end performance for the applications. The objective is to maximize the total throughput when satisfying the QoS requirements of different service classes. The proposed scheduling algorithm modifies cross-layer algorithm which incorporates SNR value and the minimum required throughput of the SS in its formulation. The SS with highest priority is selected to transmit in the frame. The priority of the SS is calculated based on the traffic class it belongs to.

\section{Algorithm:}

- Define higher priority queue

- Schedule the Bandwidth request opportunities which should be scheduled in next frame

- Periodically check the deadline for the service flow

- Do check the bandwidth minimum availability

- Resources should be periodically distributed among the service flow according to the deadline 


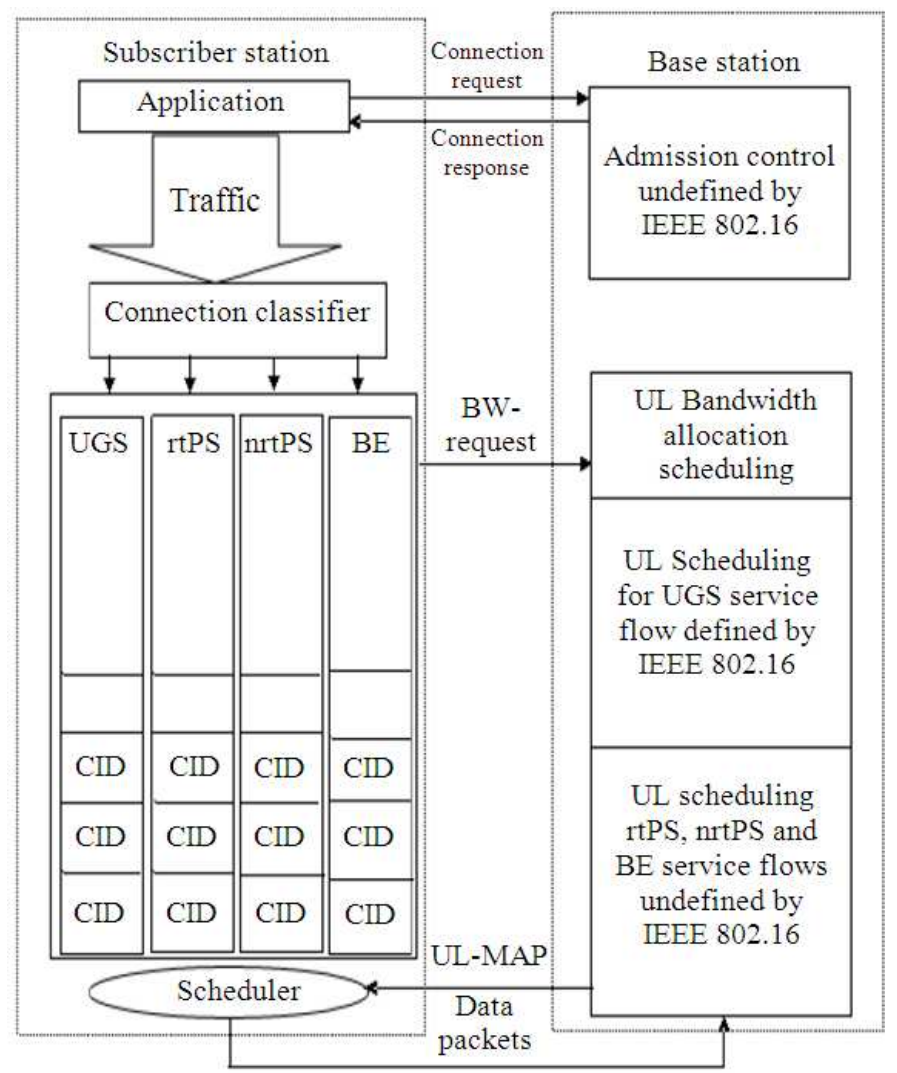

Fig. 1. IEEE 802.16 network architecture
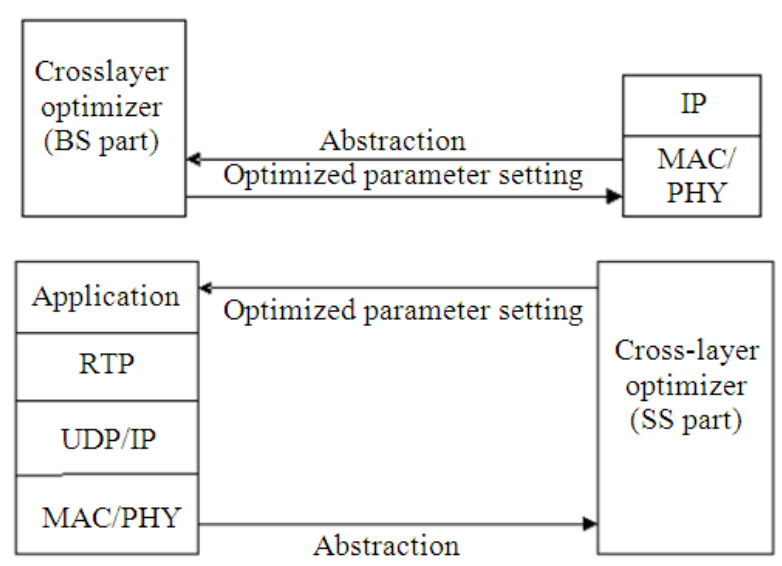

Fig. 2. Cross-layer functionality

The algorithm is executed at the BS at the beginning of every frame thereby priority is assigned to each SS. The cross layer algorithm proposed in (Elfishawy et al., 2011) implies three drawbacks. The modified cross-layer scheduling algorithm improves those drawbacks in the following ways and efficiently manages the bandwidth allocation:

- Required slots are allocated to higher priority packets and not only to one packet

- Multiple packets are in same priority, the one with earliest arrived has been picked up to decrease the delay

- Fragmentation is done for service types to make use of the available slots except the ertPS connection in WiMAX frame

Based on SNR, the type of modulation can be chosen from Table 1.

Four different buffers were used, each for one service flow. Each buffer has length $t$ and each packet received in the uplink session is stored in the buffer with the serial number, service flow identification, SNR, arrival time and packet size. The responsibility of the scheduler is to visit each buffer during the downlink subframe and to schedule the packets based on the proposed algorithm.

The flow of scheduling algorithm has been presented as flowchart for easy understanding in Fig. 3 . 


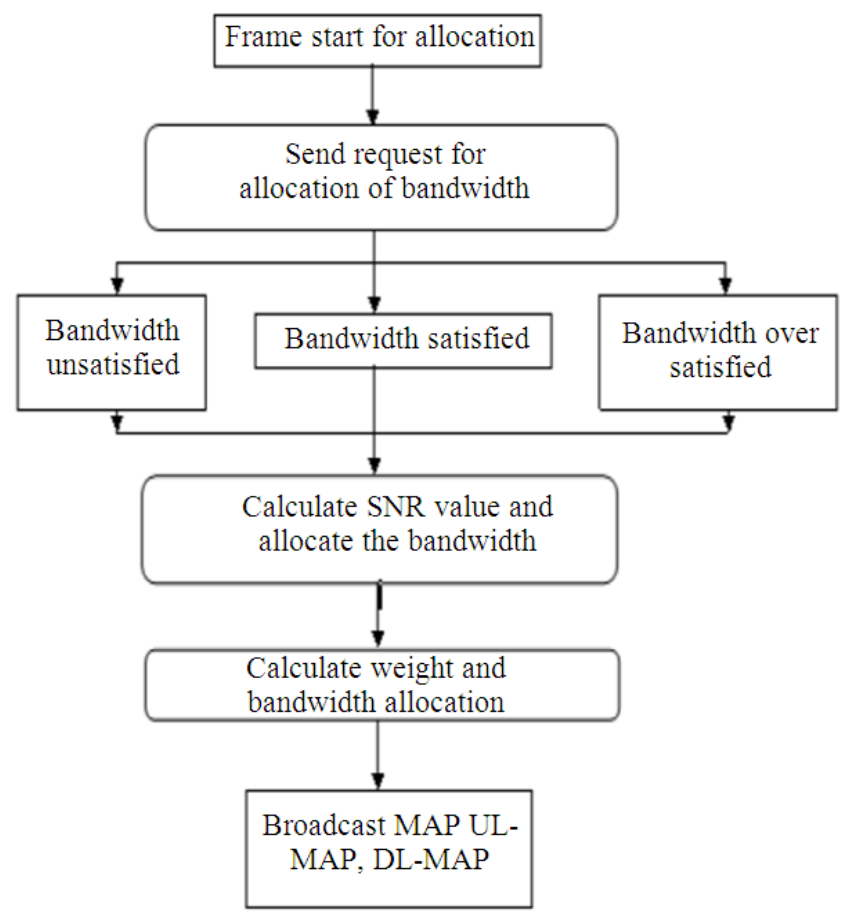

Fig. 3. Flowchart of scheduling algorithm

Table 1. MCS and receiver SNR

\begin{tabular}{lllc}
\hline S/N & Modulation & Coding rate & SNR $(\mathrm{dB})$ \\
\hline 1 & QPSK & $1 / 2$ & 5.0 \\
& & $3 / 4$ & 8.0 \\
2 & 16-QAM & $1 / 2$ & 10.5 \\
& & $3 / 4$ & 14.0 \\
3 & \multirow{2}{*}{ 64-QAM } & $1 / 2$ & 16.0 \\
& & $2 / 3$ & 18.0 \\
& & $3 / 4$ & 20.0 \\
\hline
\end{tabular}

\subsection{Simulation Methods}

The scheduler proposed in this study was implemented in the IEEE 802.16 module in NS-2 miracle simulator. The simulated network uses a point to multipoint topology (PMP) with a centralized BS and the SS. The distance between MSS and BS ranges from 1600 to 1800 meters. In our simulation, for sending the bandwidth request from all SSs, unicast polling is used. Here, the Grant per Subscriber Station (GPSS) bandwidth allocation scheme is used. In the simulation, number of calls generated by SSs is varied and is randomly generated.

The simulation parameters settings are shown in Table 2. Base station receives all transmitted packets from the subscriber stations; assigns packet serial number, packet service flow identification and arrival time and stores the packet in appropriate buffer of the service flow. Each transmitted packets have its own estimated SNR value as shown in Table 1. BS schedules the packets based on the cross-layer scheduling algorithm during the downlink session. According to the values of packet size and SNR value, required numbers of slots are allotted for each of the packets. If the required number of slots on the current frame is not enough to schedule the current packet, then the packet is lost. The buffers are used for handling different service flow. Each buffer can store 250 packets at a time. If the buffer is full and there is a packet on the queue the packet is considered to be lost since there is no memory to hold it. Once the packet is scheduled, it should be removed from the buffer and memory is considered empty to store the next packet. The uplink duration is $4.5 \mathrm{~ms}$ and the downlink duration is $5.3 \mathrm{~ms}$.

The experiment was conducted with the proposed algorithm and compared with WRR scheduling algorithm. The vital QoS parameter throughput, bandwidth efficiency, transmission efficiency is calculated for different kinds of traffic with varies number of SSs:

BW Utilization ratio $=\mathrm{R} / \mathrm{S}_{\mathrm{k}}$

Transmission efficiency $=R / S_{d}$

$\mathrm{R}=$ Total received SDU size (in bits) in BS MAC layer, total payload size which is delivered to the upper layer

$\mathrm{S}_{\mathrm{k}}=$ The total PDU size sent in SS

$S_{d}=$ The slot number for sending the PDUs 
Table 2. System parameters

\begin{tabular}{ll}
\hline Parameter & Value \\
\hline Physical layer & $\begin{array}{l}\text { Wireless MAN- } \\
\text { OFDMA,TDD }\end{array}$ \\
$\begin{array}{l}\text { No of OFDM symbols } \\
\text { and sub channels }\end{array}$ & 19,32 \\
$\begin{array}{l}\text { Bandwidth and frame } \\
\text { duration }\end{array}$ & $10 \mathrm{MHz}$ and $5 \mathrm{~ms}$ \\
$\begin{array}{l}\text { Minimum resource } \\
\text { allocation unit(slot) }\end{array}$ & $\begin{array}{l}2 \text { OFDM symbols in time, } 1 \\
\text { Max PDU size }\end{array}$ \\
\hline
\end{tabular}

Table 3. Average throughput of BS

\begin{tabular}{lllll}
\hline \multirow{2}{*}{$\begin{array}{l}\text { Total } \\
\text { Throughput } \\
\text { (kbps) }\end{array}$} & Algorithm & UGS & rtPS & \multicolumn{1}{l}{ BE } \\
\hline & Cross-Layer & 276.6667 & 278.5 & 296.1667 \\
& WRR & 236.6667 & 253.1667 & 266.6667 \\
Improved & 16.9000 & 10.0000 & 11.5300 \\
Throughput (\%) & & & \\
\hline
\end{tabular}

\subsection{Results Analysis}

To analyze the QoS in WiMAX networks, VOIP application is considered. For each of the scenario, the simulation time is $40 \mathrm{sec}$. The following simulation results are obtained based on average of 10 independent simulations presented in $95 \%$ confidence intervals. The results are analyzed between CL algorithm and conventional WRR.

For the codec scheme G.711, the number of nodes with the VOIP traffic is varied from 1, 3, 5, 7, 9 and 11. The experiment is repeated only for the following service flows defined by IEEE 802.16 e standards BE, rtPS and UGS. In the following sections, results for G.711 codec are presented. For each service flow, the CL scheduling algorithm and WRR has been compared. The above figures show the instantaneous throughput variation over simulation time. The proposed approach outperforms the traditional scheme as shown in Table $\mathbf{3}$, since the average system throughput has been enhanced almost all of the simulation time. The throughput enhancement is about $12.81 \%$ with a maximum value of $30 \mathrm{kbps}$.

The BW utilization ratio, transmission efficiency are presented in Fig. 4 and 5 in which SS number is increased from 1 to 20 . Generally WRR achieves the scheduling fairness by allocating slots to the connections based on the quantum size. If the SS are larger then more packet fragmentation will occur. This causes low bandwidth utilization ratio and low transmission efficiency. Since the cross-layer scheduling algorithm selects only qualified rtPS connections according to predefined criteria, the ULMAP overhead and MAC overhead are smaller which results in good BW utilization ratio and transmission efficiency. When number of SS is big, there is quick decrease in BW utilization ratio and transmission efficiency. A big SDU can be fragmented to many parts. If all the fragmented parts should reach at the BS MAC layer, the SDU can be defragmented successfully and delivered to the upper layer. If it is not arrived on time then fragments will be dropped in the BS MAC layer. When SS number reaches big, only part of the fragments can be successfully transmitted, therefore the received payload number decreases rapidly.

\subsection{VOIP Traffic Over BE Service Flow}

In the first case the VOIP is setup over BE service flow. Figure 6 shows the throughput for number of nodes which increase from 1 to 11 . As we can see from Fig. 6, the graph shows the better throughput of BE service flow when compared with conventional WRR scheduling algorithm.

When the number of nodes increases, the throughput also increases. This is one of the expected periods, when the number of nodes increases with the number of packets being transmitted. Each node is transmitting 180 byte packets at the rate of 60 packets per second in $G$ 711 codec scheme. The packets are generated at the rate of $64 \mathrm{kbps}$. So, the throughput for 3 nodes is around 120 kbps. For 11 nodes, the value reaches around $525 \mathrm{kbps}$. This is due to loss of packets.

\subsection{VOIP Traffic Over rtPS Service Flow}

The variation of throughput has been presented in Fig. 7 where VOIP traffic over rtPS service flow is carried over. G.711 codec scheme is used for VOIP. Similar to the Best Effort service flow case, the throughput increases steadily as the number of nodes increases. The throughput is $116 \mathrm{kbps}$ for 3 nodes and goes up to $520 \mathrm{kbps}$ for 11 nodes. As the figure shows the performance of cross-layer algorithm consistently outperforms the conventional round robin scheduling algorithm.

\subsection{VOIP Traffic for UGS Service Flow}

Figure 8 shows the variation of throughput for VOIP traffic over UGS service flow. Again there is a steady increase when the number of nodes increases. The values range from $120 \mathrm{kbps}$ for 3 nodes $580 \mathrm{kbps}$ for 11 nodes. From the above figure it is proved that the throughput of $\mathrm{CL}$ algorithm is better than WRR algorithm. 
R. Nandhini and N. Devarajan / Journal of Computer Science 10 (2): 255-263, 2014

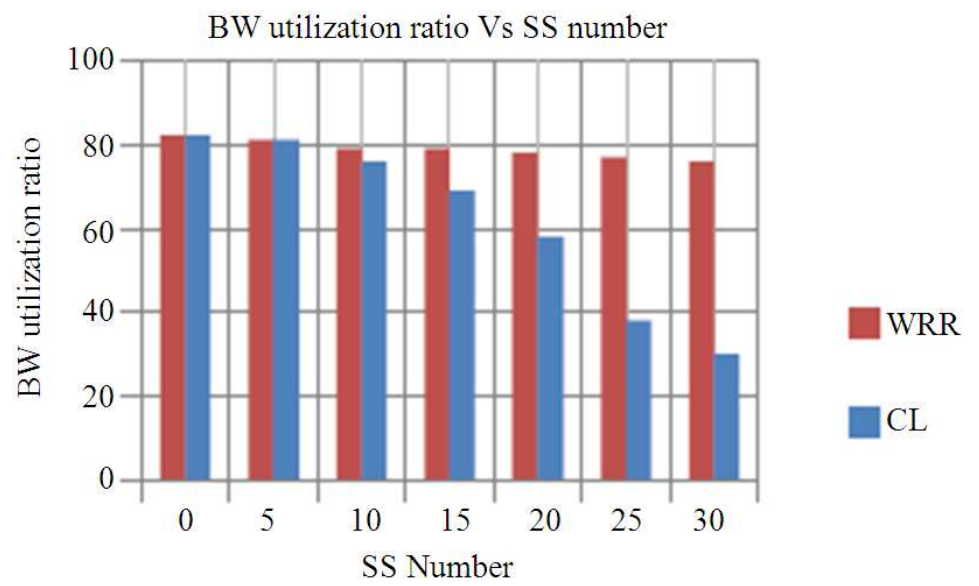

Fig. 4. BW utilization versus SS number

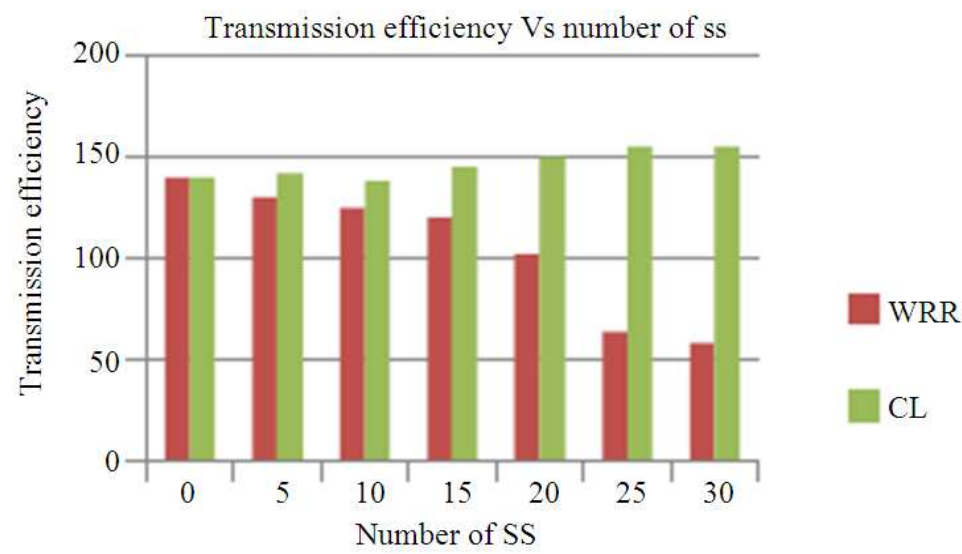

Fig. 5. Transmission efficiency versus SS number

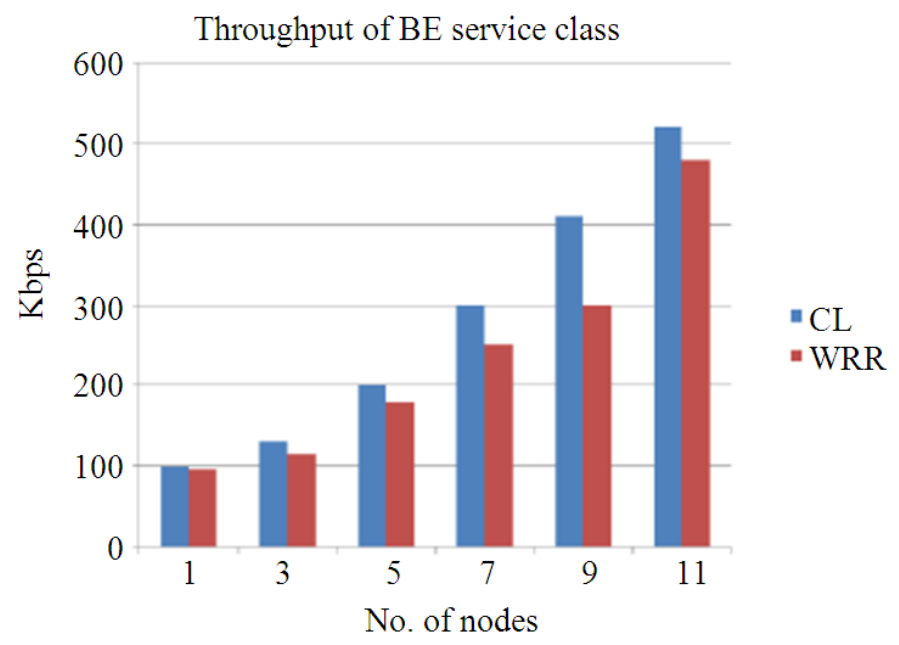

Fig. 6. Throughput with the number of nodes for BE service flow 


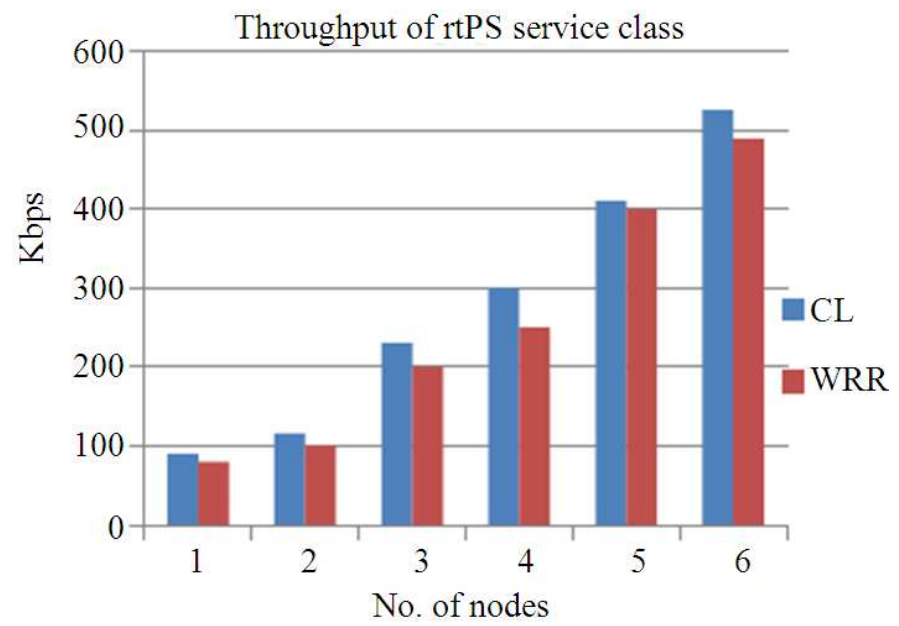

Fig. 7. Throughput with the number of nodes for rtPS service flow

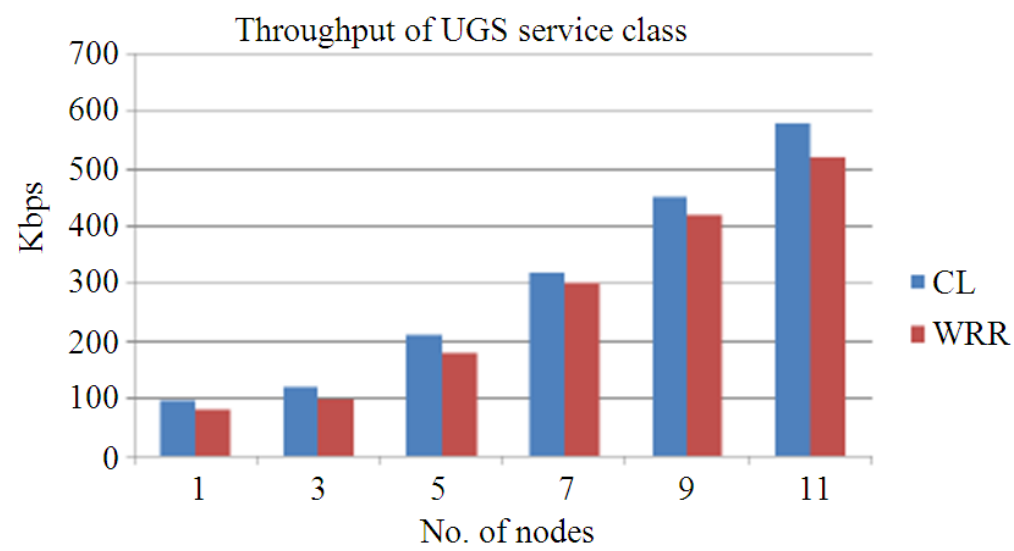

Fig. 8. Throughput with the number of nodes for UGS service flow

\section{CONCLUSION}

In this study, a cross-layer QoS based scheduling algorithm has been compared with WRR in WiMAX using the NS2 simulator. These simulations describe the behavior of both algorithms to respect the QoS of each service class. The studied parameters have a direct influence on the flows nature throughput and transmission efficiency and bandwidth utilization. The WRR algorithm is easy to be implemented in hardware. A cross-layer scheduling algorithm which aims at providing improved performance and guaranteed throughput by $12.8 \%$ and QoS requirements has been proposed. The performance improvement of the proposed scheme is illustrated through the simulation results. In this work, static IEEE 802.16 network is considered. In the future work, subscriber mobility will be considered and more codec schemes for VOIP will be taken for more real-time operating environment.

\section{REFERENCES}

Borin, J.F. and N.LS.D. Fonseca, 2009. Uplink scheduler and admission control for the IEEE 802.16 standard. Proceedings of the IEEE Global Telecommunications Conference, Nov. 30-Dec. 04, IEEE Xplore Press, Honolulu, HI., pp: 1-6. DOI: 10.1109/GLOCOM.2009.5425779

Chandra, G. and Praveen, 2009. Tata consultancy services. 
El-fishawy, N.A., M. Zahra, M. Ebrahim and M.M. Elgamala, 2011. Modified cross-layer scheduling for mobile WiMAX networks. Proceedings of the 28th National Radio Science Conference (NRSC), Apr. 26-28, IEEE Xplore Press, Cairo, pp: 1-10. DOI: 10.1109/NRSC.2011.5873602

Ghazizzadeh, R., P. Fan and Y. Pan, 2009. A two layer channel aware scheduling algorithm for IEEE 802.16 broadband wireless access systems. J. Applied Sci., 9: 449-458. DOI: 10.3923/jas.2009.449.458

Lu, J. and M. Ma, 2011. Cross-layer QoS support framework and holistic opportunistic scheduling for QoS in single carrier WiMAX system. J. Netw. Comput. Applic., 34: 765-773. DOI: 10.1016/j.jnca.2010.10.005
Revankar, P.S., A.S. Kapse and W.Z. Gandhare, 2010. Overview of MAC scheduling algorithm for IEEE 802.16 wireless networks. Int. J. Comput. Appli., 2: 5-8. DOI: 10.5120/689-968

Shuaibu, D.S., S.K. Syed-yus of and N. Fisal, 2010. A cross layer approach for packet scheduling at downlink of WiMAX IEEE802.16e. Eur. J. Sci. Res., 45: 529-539.

Wu, S.J., S.Y. Huang and K.F. Huang, 2012. Efficient quality of service scheduling mechanism for WiMAX networks. Comput. Commun., 35: 936951. DOI: 10.1016/j.comcom.2012.02.002 